\title{
DYNAMIC MODELLING AND ANALYZING OF A WALKING OF HUMANOID ROBOT
}

\author{
BAJRAMI Xhevahir ${ }^{1}$, SHALA Ahmet ${ }^{1}$, HOXHA Gezim ${ }^{1}$, LIKAJ Rame ${ }^{1}$ \\ ${ }^{1}$ University of Prishtina "Hasan Prishtina", Faculty of Mechanical Engineering, Department of Mechatronics, \\ Pristina, Bregu i Diellit, p.n.10000 Prishtinë, Kosovës, e- mail: gezim.hoxha@uni-pr.edu
}

\begin{abstract}
This paper focuses on the walking improvement of a biped robot. The zero-moment point (ZMP) method is used to stabilise the walking process of robot. The kinematic model of the humanoid robot is based on DenavitHartenberg's (D-H) method, as presented in this paper. This work deals with the stability analysis of a two-legged robot during double and single foot walking. It seems more difficult to analyse the dynamic behaviour of a walking robot due to its mathematical complexity. In this context most humanoid robots are based on the control model. This method needs to design not only a model of the robot itself but also the surrounding environment. In this paper, a kinematic simulation of the robotic system is performed in MATLAB. Driving torque of the left and right ankle is calculated based on the trajectory of joint angle, the same as angular velocity and angular acceleration. During this process an elmo motion controller is used for all joints. The validity of the dynamic model is tested by comparing obtained results with the simulation results.
\end{abstract}

KEYWORDS: D-H conversation; kinematics; dynamics; walking motion; humanoid robot; Simechanics.

\section{Introduction}

SimMechanics has a number of physical component blocks, such as bodies, joints, constraints, coordinate systems, actuators, sensors, and so on. SimMechanics provides a variety of simulation and analysis modes for mechanical systems:

- Forward Dynamic Analysis solves the response to given excitation of the mechanical system;

- Reverse Dynamic Analysis solves the required force and torque according to the results of a given movement of the mechanical system;

- Kinematic Analysis solves the system's displacement, velocity and acceleration under constraint conditions, and checks the consistency;

- Linear Analysis obtains the linear model of the system in the designation of small perturbation or initial state to analyse the system's response performance;

- Equilibrium Point Analysis determines the steady-state equilibrium point for linear system analysis [5].

\section{Gait Analysis}

Gait analysis is the study of animal locomotion, including locomotion of humans [9]. Describing human gait requires some specific terms, which are defined in this section. The gait cycle begins when one foot contacts the ground and ends when that foot contacts the ground again. Thus, each cycle begins at initial contact with a stance phase and proceeds through a swing phase until the cycle ends with the limb's next initial contact [1], [2], [3], [12], [16]. 


\section{Motion planning OXYZ}

In this section, planning motion for the $3 \mathrm{~d}$ (axes $\mathrm{x}, \mathrm{y}, \mathrm{z}$ ) is accomplished whilst considering two the phases of double support and single support and considering that the transition between the foot support is instantaneous [12]. To accomplish this some tasks required certain parameters as they determine the walking configuration, as shown in Fig. 1.

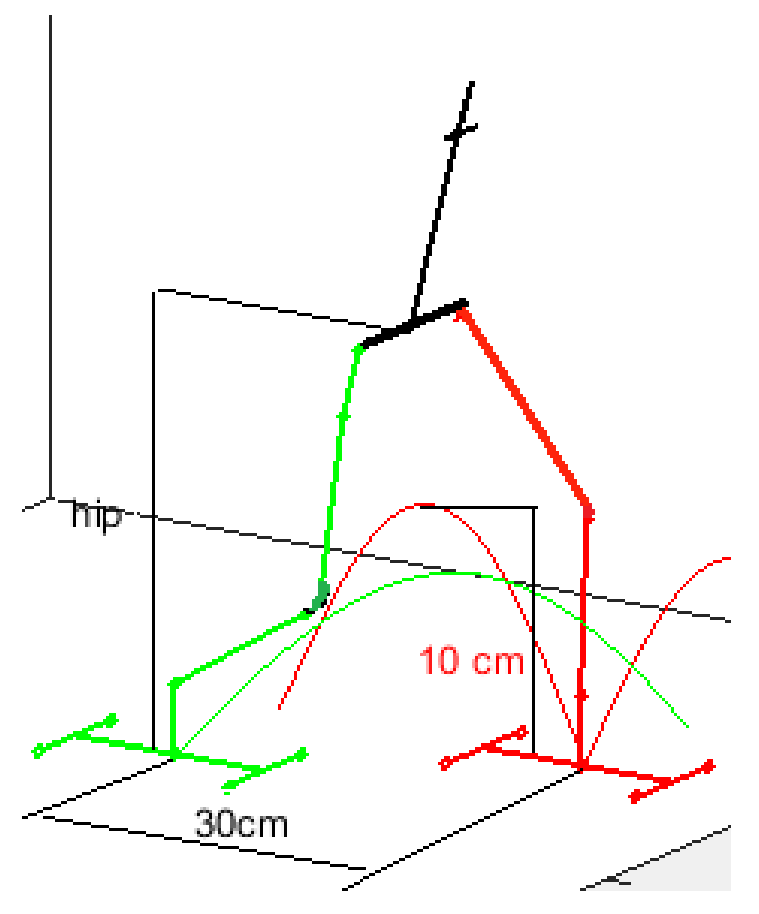

Fig. 1 Coordinates for planning motion in the 3D

Where the explanations below for abbreviations used in Fig. 1: $\mathrm{T}_{\mathrm{s}}-$ time for each step, $\mathrm{SL}=30$ - length of step, $\mathrm{h}_{\text {hip }}$ - twisting of the hip, $\mathrm{H}_{\mathrm{m}}=10$ - height of the floating leg.

\section{$4 \quad$ Trajectories of the swing limb}

The trajectory of the tip of the swing limb during the SSP is an important factor in biped walking [12]. In this section, we develop constraint equations that can be used for solving the swing limb trajectory.

The trajectory of the tip of the swing limb is denoted by the vector $\mathrm{X}_{\mathrm{a}}:\left(\mathrm{x}_{\mathrm{a}}(\mathrm{t}), \mathrm{y}_{\mathrm{a}}(\mathrm{t})\right)$, where $\left(x_{a}(t), y_{a}(t)\right)$ is the coordinate of the swing limb tip position with the origin of the coordinate system located at the tip of the supporting limb. We use a third order polynomial and a fifth order polynomial functions for the $\mathrm{x}_{\mathrm{a}}$ and $\mathrm{y}_{\mathrm{a}}$ separately [12].

$$
x_{a}(t)=\left\{\begin{array}{l}
x_{a}=a_{0}+\ldots+a_{3}(k) \cdot t^{3} \\
y_{a}=b_{0}+b_{1} \cdot t+\ldots+b_{5}(k) \cdot t^{5}
\end{array} \quad 0<t<T_{s}\right.
$$

Next, the constraint equations that can be used for solving the coefficients, $a_{i}$ and $b_{j}(i=$ $0 \ldots 3$ and $j=0 \ldots 5$ ) are derived. Casting the gait patterns in terms of four basic quantities: step length $S_{L}$, step period for the SSP $T_{S}$, maximum clearance of the swing limb $\mathrm{H}_{\mathrm{m}}$, and its location $\mathrm{S}_{\mathrm{m}}$. Other constraints used for designing the swing limb motion are repeatable gait and minimising the effect of impact. The constraint relations are described as follows: 
* Geometrical constraints: The swing limb has to be lifted off the ground at the beginning of the step and has to land back at the end. We will enforce this condition by the following equation [12]:

$$
y_{a}(0)=0 \text { and } y_{a}\left(T_{s}\right)=0
$$

* Maximum clearance of the swing limb: During the swing phase, the tip of the swing limb has to stay clear off the ground to avoid accidental contact. In some previous work, the parabolic relation between $\mathrm{x}_{\mathrm{a}}(\mathrm{t})$, and $\mathrm{y}_{\mathrm{a}}(\mathrm{t})$, has been assumed [12]. Although this strategy has the advantage of being the simplest form that allows the prescription of the desired step length and the tip maximum clearance independently, it is unlikely satisfies the requirement of repeatable gait. In this work, we synthesize the swing limb trajectory by setting the following relation [12]:

$$
x_{a}\left(T_{M}\right)=S_{M}, y_{a}\left(T_{m}\right)=H_{M} \text { and } \frac{d}{d t}\left(y_{a}\left(T_{m}\right)\right)=0
$$

where, $\mathrm{H}_{\mathrm{m}}=0.05[\mathrm{~m}]$ is the maximum clearance of the swing limb, $\mathrm{S}_{\mathrm{m}}=0[\mathrm{~m}]$ is the $\mathrm{x}$ coordinate of the swing limb tip corresponding to the maximum clearance, and $\mathrm{T}_{\mathrm{m}}$ is the time instant when the tip of the swing limb reaches to the maximum clearance.

* Repeatability of the gait: The requirement for repeatable gait imposes the initial posture and angular velocities to be identical to those at the end of the step [12]. Furthermore, since during the DSP both tips are in contact with the ground and remain stationary, the initial velocities in both horizontal and vertical direction must remain zero. Subsequently, the following relations must hold [12]:

$$
\left.x_{a}(k)=-\frac{S_{L}(k)}{2}, x_{a}\left(T_{S}\right)=\frac{S_{L}(k)}{2}, \frac{d}{d t}\left(x_{a}\left(k+T_{S}\right)\right)=0\right\}
$$

where: $K(k)$ and $S_{L}(k)$ representation matrices of the trajectory of feet.

$$
\begin{gathered}
\left.K(k)=\| \begin{array}{cccccc}
T_{m}^{2} & T_{m}^{3} & 0 & 0 & 0 & 0 \\
0 & 0 & T_{s}^{2} & T_{s}^{3} & T_{s}^{4} & T_{s}^{5} \\
0 & 0 & T_{m}^{2} & T_{m}^{3} & T_{m}^{4} & T_{m}^{5} \\
0 & 0 & 2 T_{m} & 3 T_{m}^{2} & 4 T_{m}^{3} & 5 T_{m}^{4} \\
T_{s}^{2} & T_{s}^{3} & 0 & 0 & 0 & 0 \\
0 & 0 & 2 T_{s} & 3 T_{s}^{2} & 4 T_{s}^{3} & 5 T_{s}^{4}
\end{array}\right] \cdot\left[\begin{array}{c}
S_{m}-a_{o}(k)-a_{1} \cdot T_{m} \\
-b_{o}-b_{1} \cdot T_{s} \\
H_{m}-b_{o}-b_{1} \cdot T_{s} \\
-b_{1} \\
\frac{S_{L}(k)}{2}-a_{o}(k)-a_{1} \cdot T_{s} \\
-a_{1}
\end{array}\right] \\
S_{L}(k)=\left\|\begin{array}{ccc}
0.7 & \text { if } \quad 0 \leq k \leq 0.7 \\
0.6 & \text { if } \quad 0 \leq k \leq 1.4 \\
0.6 & \text { if } \quad 0 \leq k \leq 1.4 \\
0.7 \quad \text { if } \quad 9.7 \leq k \leq 10.5
\end{array}\right\| \\
a_{0}(k)=-\frac{S_{L}(k)}{2}, \quad S_{m}=0, T_{m}=\frac{T_{s}}{2}
\end{gathered}
$$

From equation (1) variables are derived as follows: 
Table 1: Optimisation parameters for foot trajectory

\begin{tabular}{|c|c|c|c|l|l|}
\hline \multicolumn{7}{|c|}{ Parameters } \\
\hline $\boldsymbol{a}_{\mathbf{0}}$ & $\boldsymbol{a}_{\mathbf{1}}$ & $\boldsymbol{a}_{\mathbf{2}}$ & $\boldsymbol{a}_{3}$ & \multicolumn{1}{|}{} \\
\hline-0.35 & 0 & 5.833 & -6.481 & & \\
\hline $\boldsymbol{b}_{\mathbf{0}}$ & $\boldsymbol{b}_{\boldsymbol{1}}$ & $\boldsymbol{b}_{\mathbf{2}}$ & $\boldsymbol{b}_{\mathbf{3}}$ & $\boldsymbol{b}_{\mathbf{4}}$ & $\boldsymbol{b}_{5}$ \\
\hline 0 & 0 & 8.889 & -29.63 & 24.691 & $1.645 \times 10^{-13}$ \\
\hline
\end{tabular}

\section{Trajectories of the hip}

Hip motion has a significant effect on the stability of the biped system [12]. Here, the trajectory of the hip is designed for the SSP and the DSP separately, which are denoted by the coordinate of the hip position as $X_{h S}:\left(x_{h S}(t), y_{h S}(t)\right)$ in the SSP, and $X_{h D}:\left(x_{h D}(t), y_{h D}(t)\right)$ in the DSP. A third order polynomial function is used to describe $x_{h s}$ and $x_{h D}$, respectively. With a general function of vertical hip motion, they are shown as below [12]:

$$
\left.\begin{array}{c}
x_{h s}(t)=c_{0}(k)+c_{1} \cdot t+c_{2}(k) \cdot t^{2}+c_{3}(k) \cdot t^{3}, \quad T_{s}<t \leq T_{D} \\
x_{h D}(t)=d_{0}(k)+d_{1}(k) \cdot t_{D}+d_{2}(k) \cdot t_{D}^{2}+d_{3}(k) \cdot t_{D}^{3}, \quad T_{s}<t_{D} \leq T_{D}
\end{array}\right\}
$$

Next, we develop constraint equations which include the additional quantities: positions of the hip at the beginning of the SSP and the DSP, $S_{S O}$ and $S_{D O}$, step period for the DSP, $T_{D}$, and the height of the hip, $H_{h}$. Besides the constraints of repeatable gait and minimising the effect of impact, the stability of the biped walking during the DSP is also considered. The constraint relations are described as follows [12]:

- Vertical hip motion:

$$
\left.\begin{array}{l}
y_{h S}(t)=H_{h} \\
y_{D S}(t)=H_{h}
\end{array}\right\}
$$

$H_{h}$ should be given such that the robot does not go through the singular configurations.

* Repeatability of the gait: To keep the gait repeatable, the posture and angular velocity at the beginning of the SSP must be identical to that at the end of the DSP. Thus, the following relations must hold [12].

$$
\left.\begin{array}{c}
x_{h S}(0)=-S_{S O} \\
x_{h D}(k)=\frac{S_{L}(k)}{2}-S_{S O}
\end{array}\right\}
$$

Where: $x h_{0}(k)=\frac{-S_{L}(k)}{4}, S_{s 0}(k)=\left|x_{a}(0, k)-x h_{0}(k)\right|$

Variables that change, if: $S D_{0}(k)=\frac{S_{L}(k)}{2}-0.2$, then with $c_{0}(k)=-S_{s 0}(k), c_{1}=v_{h 1}$. Follows 


$$
\left.K h(k)=\left[\begin{array}{cccccc}
T_{s}^{2} & T_{s}^{3} & 0 & 0 & 0 & 0 \\
2 T_{s} & 3 T_{s}^{2} & 0 & 0 & 0 & 0 \\
0 & 0 & 1 & T_{s} & T_{s}^{2} & T_{s}^{3} \\
0 & 0 & 1 & T_{D} & T_{D}^{2} & T_{D}^{3} \\
0 & 0 & 0 & 1 & 2 T_{s} & 3 T_{s}^{2} \\
0 & 0 & 0 & 1 & 2 T_{D} & 3 T_{D}^{2}
\end{array}\right]^{-1}\left[\begin{array}{c}
S_{D O}(k)-\left(c_{o}(k)-a_{1} \cdot T_{s}\right) \\
V_{h 2}-c_{1} \\
S_{D O}(k) \\
\frac{S_{L}(k)}{2}-S_{S O}(k) \\
V_{h 2} \\
V_{h 1}
\end{array}\right]\right\}
$$

Where: $K h(k)$ representation matrices of the trajectory of hip.

* Continuity of the gait: The hip trajectory must be continuous during the whole gait cycle, i.e., the horizontal displacements and velocities of the hip at the end of the SSP and the beginning of the DSP must be identical respectively, which leads to [12]:

$$
\begin{aligned}
& x_{h D}(0)=S_{D O} \\
& \left.x_{h S}(k)=S_{D O}\right\} \\
& \left.\begin{array}{l}
\frac{d}{d t}\left(x_{h D}(0)\right)=V_{h 2} \\
\frac{d}{d t}\left(x_{h S}(k)\right)=V_{h 2}
\end{array}\right\}
\end{aligned}
$$

Equations (7) and (8) follows for the variables $c_{0}(k), c_{1}(k), c_{2}(k), c_{3}(k), d_{0}(k), d_{1}(k)$, $d_{2}(k), d_{3}(k)$ and are presented below with the respective values:

Table 2: Optimisation parameters for hip trajectory

\begin{tabular}{|c|c|c|c|}
\hline \multicolumn{4}{|c|}{ Parameters } \\
\hline $\boldsymbol{c}_{\boldsymbol{0}}$ & $\boldsymbol{c}_{\boldsymbol{1}}$ & $\boldsymbol{c}_{\boldsymbol{2}}$ & $\boldsymbol{c}_{\boldsymbol{3}}$ \\
\hline-0.175 & 0.5 & 0.958 & -1.481 \\
\hline $\boldsymbol{d}_{\boldsymbol{0}}$ & $\boldsymbol{d}_{\boldsymbol{1}}$ & $\boldsymbol{d}_{\boldsymbol{2}}$ & $\boldsymbol{d}_{\boldsymbol{3}}$ \\
\hline-0.42 & 3.65 & -7.5 & 5 \\
\hline
\end{tabular}

Where: xa1, xa2, ya1, ya2 are trajectories of foots on x respectively y-axes

$$
\begin{aligned}
& x_{a 1}=\mid \begin{array}{c}
x_{a}(t, k) \quad \text { if } \quad 0<t \leq s \\
x_{a}(s, k) \text { if } \quad s<t \leq 2 s+2 d \\
{\left[x_{a}[t-(2 s+2 d), 2 k]+x_{a 1}(2 s+2 d)\right.} \\
\left.-x_{a}(0,2 k)\right] \text { if } \quad 2 s+2 d<t \leq 3 s+2 d
\end{array} \\
& x_{a 2}=\mid \begin{array}{c}
0 \text { if } 0<t \leq s+d \\
x_{a}[t-(s+d), 2 k]-x_{a}(0,2 k] \quad \text { if } \quad s+d<t \leq 2 s+d \\
{\left[x_{a 2}(2 s+d) \text { if } 2 s+d<t \leq 3 s+3 d\right.}
\end{array} \\
& y_{a 1}=\mid \begin{array}{rll}
y_{a}(t, k) & \text { if } \quad 0<t \leq s \\
0 & \text { if } \quad s<t \leq 2 s+2 d \\
{\left[y_{a}[t-(2 s+2 d), 2 k] \quad \text { if } \quad 2 s+2 d<t \leq 3 s+2 d\right.}
\end{array}
\end{aligned}
$$




$$
\begin{aligned}
& y_{a 2}=\mid \begin{array}{r}
0 \text { if } \quad 0<t \leq s+d \\
y_{a}[t-(s+d), 2 k] \text { if } \quad s+d<t \leq 2 s+d \\
0 \text { if } \quad 2 s+d<t \leq 3 s+3 d
\end{array} \\
& x_{h}=\mid \begin{array}{c}
x_{h s}(t, k) \text { if } 0<t \leq s \\
x_{h D}(t, k) \text { if } s<t \leq s+d \\
x_{h s}[t-(s+d), 2 k]+x_{h}(s+d)-x_{h s}(0,2 k) \text { if } s+d<t \leq 2 s+d \\
x_{h D}[t-(s+d), 2 k]+x_{h}(1.3)-x_{h D}(s, 2 k) \text { if } 2 s+d<t \leq 2 s+2 d \\
x_{h s}[t-(2 s+2 d+0.01), 3 k]+x_{h}(2 s+2 d)-x_{h D}(0,3 k) \text { if } 2 s+2 d<t \leq 3 s+2 d
\end{array}
\end{aligned}
$$

Table 3: Optimal parameters for the foots

\begin{tabular}{|c|c|c|c|c|c|c|c|}
\hline \multicolumn{7}{|c|}{ Parameters } \\
\hline $\boldsymbol{x} \boldsymbol{a}_{1}$ & -0.35 & -0.29 & -0.16 & 0 & 0.16 & 0.29 & 0.35 \\
\hline $\boldsymbol{x} \boldsymbol{a}_{2}$ & 0.04 & 0.16 & 0.3 & 0.45 & 0.56 & 0.6 & 0.6 \\
\hline $\boldsymbol{y} \boldsymbol{a}_{1}$ & 0 & 0.062 & 0.16 & 0.2 & 0.16 & 0.062 & 0 \\
\hline $\boldsymbol{y} \boldsymbol{a}_{2}$ & 0.062 & 0.16 & 0.2 & 0.16 & 0.062 & 0 & 0 \\
\hline
\end{tabular}

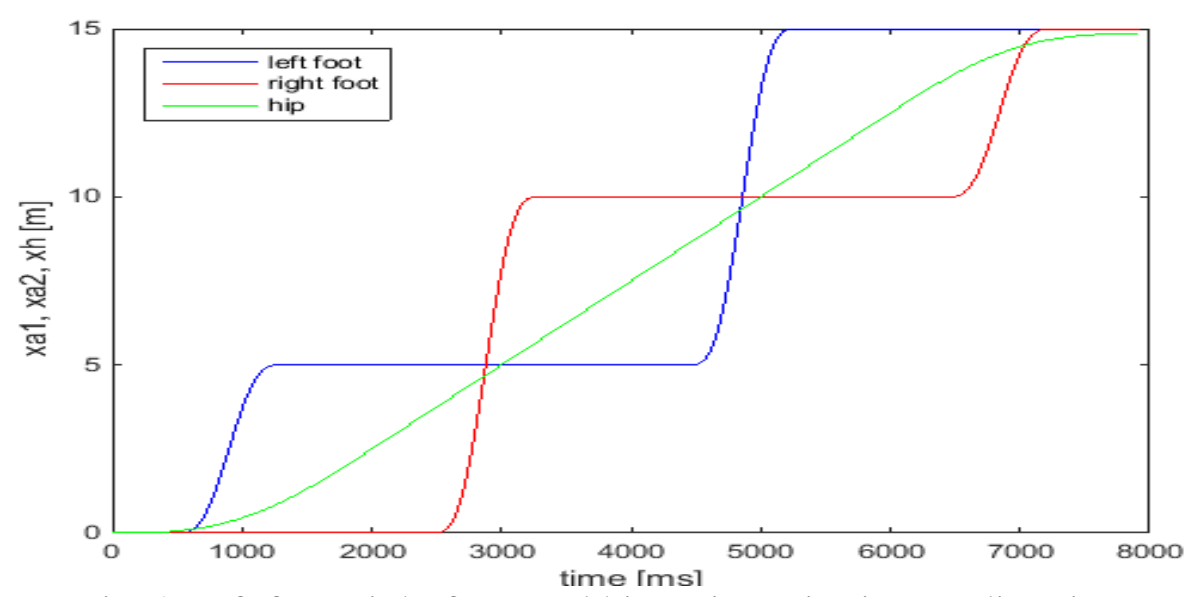

Fig. 2 Left foot, right foot, and hip trajectories in XY direction

Fig. 3 is the stick diagram of the biped walking on level ground. From this diagram, one can observe the overall motion of the biped during both the SSP and the DSP. The solid lines represent the SSP while the dashed lines represent the DSP.

The asterisk shows the Center of Gravity $(\mathrm{CoG})$ of the biped during walking. The posture of the biped at the end of each step is close to that at the beginning of each step, and the displacement of $\mathrm{CoG}$ trajectory is almost horizontal. 


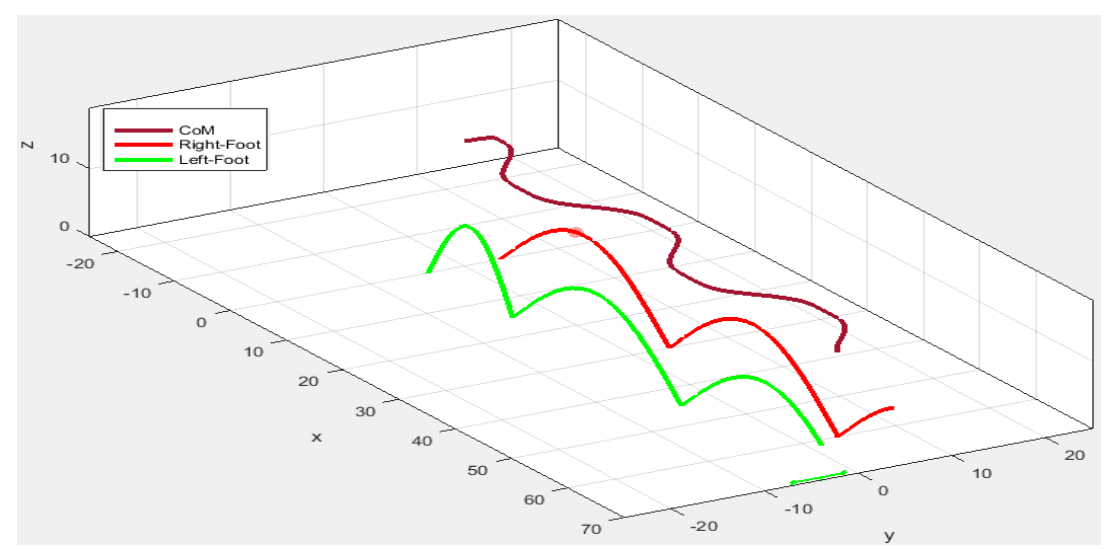

Fig. 3 Centre of Gravity pattern (brawn line), right foot (blue line), and left foot (red line)

For $\mathrm{H}_{\mathrm{m}}=0.10[\mathrm{~m}]$ - (height of the following leg) is the maximum clearance of the swing limb, $S_{m}=0[\mathrm{~m}]$ is the $\mathrm{x}$-coordinate of the swing limb tip corresponding to the maximum clearance, and $\mathrm{T}_{\mathrm{m}}$ is the time instant when the tip of the swing limb reaches the maximum clearance. The $\mathrm{CoG}$ of humanoid robotics is approximately $55 \mathrm{~cm}$ in height. Improving the legs- height during walking should lead to more stability for the robot. Through observations we can conclude that this can be achieved by adding mass, which means reducing height of the $\mathrm{CoG}$ (for example, $3 \mathrm{~kg}$ batteries placed under the hip), in proportion with the motor force. The main aim is to bring the centre of gravity as near to the ground as possible which means that all heavy parts should be mounted as low as possible.

The feet are raised by $10 \mathrm{~cm}$ while maintaining stability and increasing performance. The robot foot and knee trajectory simulation are with the following values. For given step height $\mathrm{Hm}=0.15[\mathrm{~m}]$ and step length $\mathrm{So}=0.15[\mathrm{~m}]$ it is shown that maximum of step height cannot overcome the value 0.10 [m]. The overcoming of this value can lead to instability during walking.

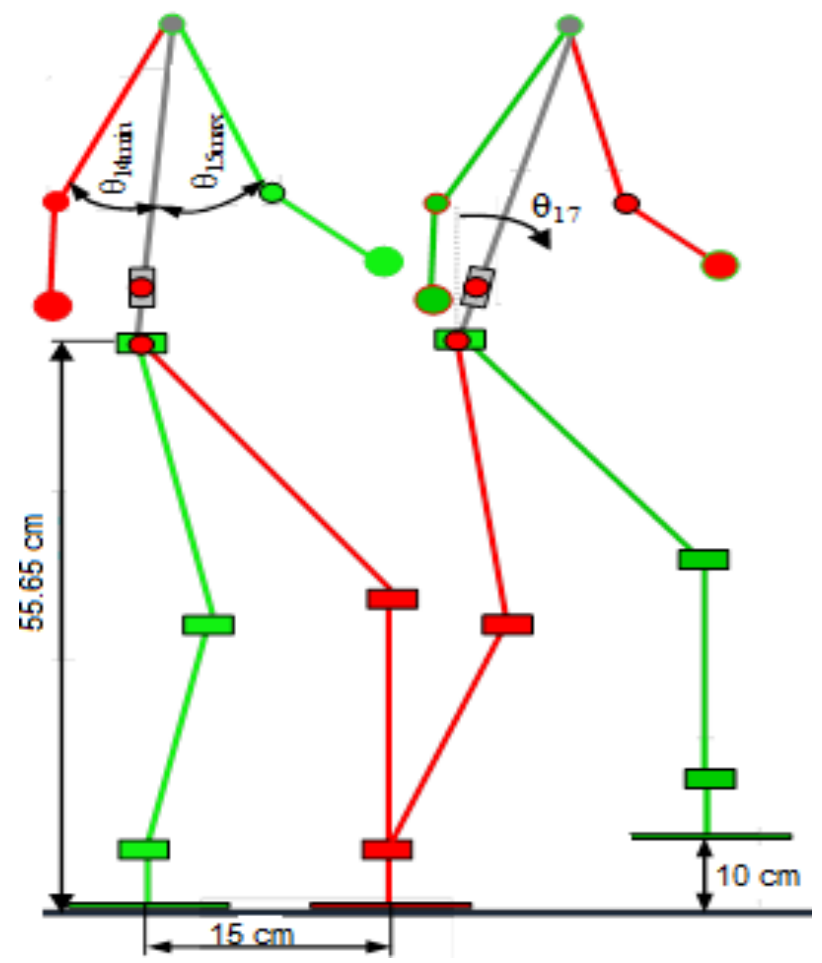

Fig. 4 Step height and step length with minimal hip 


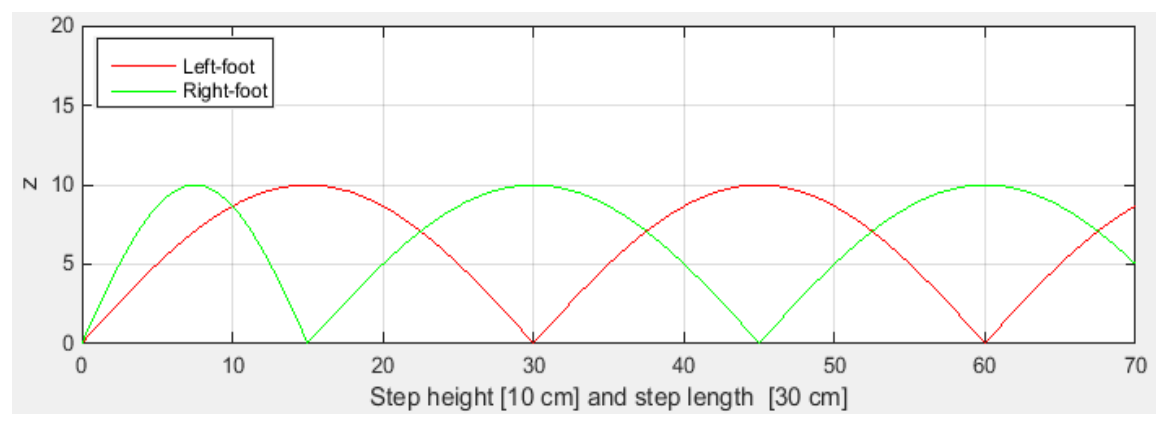

Fig. 5 Trajectory of feet for step height and step length

To determine the optimal trajectories, they are simulated using the kinematic-dynamic model. The result of each of the simulations is a set of numbers defining the stability margin for each simulation time step. The trajectory with the highest stability margin at the most critical point is chosen as the optimal trajectory. To obtain a useful trajectory it is necessary to include the physical limitations of the robot in the trajectory simulations. The physical angular limitations were determined by moving the joints one at a time to their physical limit and then performing a reading of the angle.

\section{$6 \quad$ Kinematics}

Humanoid locomotion requires an accurate forward and inverse kinematic model in order to specify desired joint angles related to the base and effectors trajectories [10].

\section{$7 \quad$ New proposed kinematic of our model}

A commonly used convention for selecting frames of reference in robotic applications is the $\mathrm{D}-\mathrm{H}$. In this convention, each homogeneous transformation $\mathrm{Ai}$ is represented as a product of four basic transformations (see Fig.6 and Table 4 below).

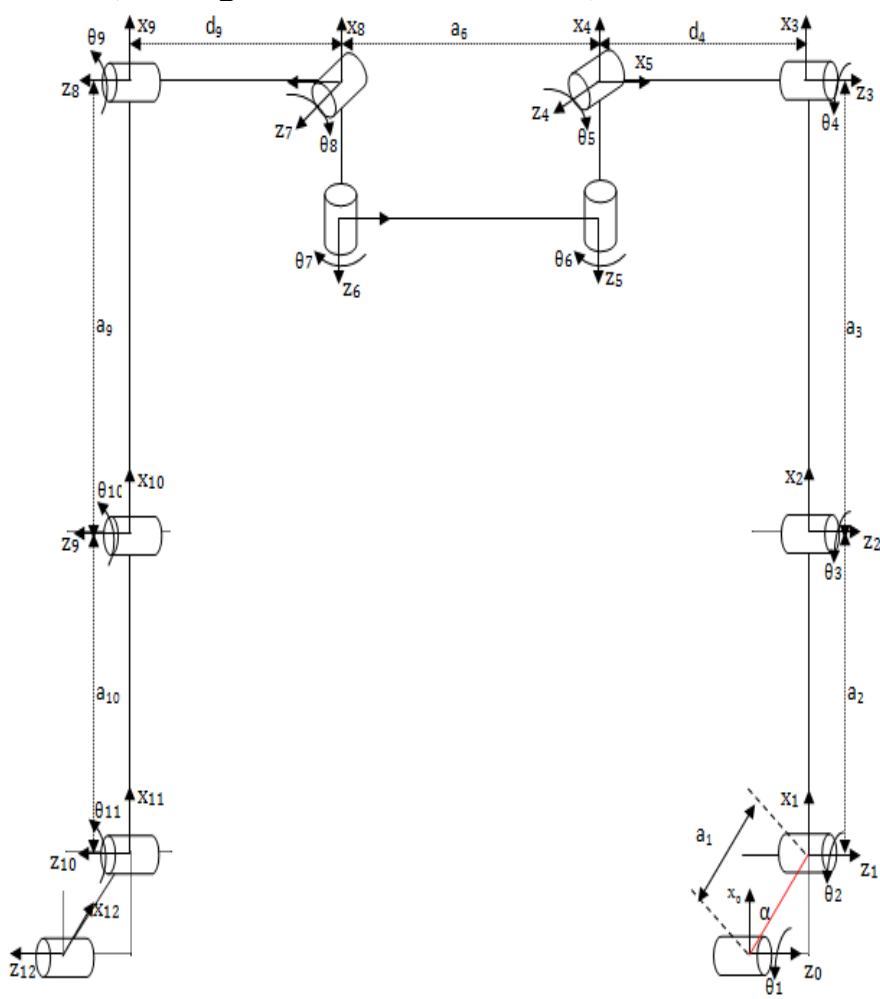

Fig. 6 Kinematic model of humanoid robot 
The Denavit-Hartenberg parameters for the 11 DOF of the lower body (derived from Fig. 6) are shown in Table 4 and can be used further for forward and inverse kinematic.

Table 4: D-H parameters for 11 DOF lower body

\begin{tabular}{|c|c|c|c|c|}
\hline Link & ai $[\mathrm{cm}]$ & $\alpha i$ degree] & di [cm] & $\theta \mathrm{i}$ [degree] \\
\hline 1 & $a_{1}$ & 0 & 0 & $\theta_{1}+\pi / 2$ \\
\hline 2 & $a_{2}$ & 0 & 0 & $\theta_{2}$ \\
\hline 3 & $a_{3}$ & 0 & 0 & $\theta_{3}$ \\
\hline 4 & 0 & $-\pi / 2$ & $-d_{4}$ & $\theta_{4}+\pi / 2$ \\
\hline 5 & 0 & $\pi / 2$ & 0 & $\theta_{5}^{*}+\pi / 2$ \\
\hline 6 & $a_{6}$ & 0 & 0 & $\theta_{6}^{*}$ \\
\hline 7 & 0 & $\pi / 2$ & 0 & $\theta_{7}^{*}+\pi / 2$ \\
\hline 8 & 0 & $-\pi / 2$ & 0 & $\theta_{8}^{*}+\pi / 2$ \\
\hline 9 & $-a_{9}$ & 0 & $d_{9}$ & $\theta_{9}^{*}$ \\
\hline 10 & $-a_{10}$ & 0 & 0 & $\theta_{10}^{*}$ \\
\hline 11 & $-a_{11}$ & 0 & 0 & $\theta_{11}^{*}+\pi / 2$ \\
\hline
\end{tabular}

The Denavit-Hartenberg transformation matrix for adjacent coordinate frames, $\mathrm{i}$ and $\mathrm{i}-1$.

$$
A_{i}=\left[\begin{array}{cccc}
\cos \left(\theta_{i}\right) & -\sin \left(\theta_{i}\right) \cdot \cos \left(\alpha_{i}\right) & \sin \left(\theta_{i}\right) \cdot \sin \left(\alpha_{i}\right) & a_{i} \cdot \cos \left(\theta_{i}\right) \\
\sin \left(\theta_{i}\right) & \cos \left(\theta_{i}\right) \cdot \cos \left(\alpha_{i}\right) & -\cos \left(\theta_{i}\right) \cdot \sin \left(\alpha_{i}\right) & a_{i} \cdot \sin \left(\theta_{i}\right) \\
0 & \sin \left(\alpha_{i}\right) & \cos \left(\alpha_{i}\right) & d_{i} \\
0 & 0 & 0 & 1
\end{array}\right]
$$

For $\mathrm{i}=1,2,3, \ldots 11$, joint $\mathrm{O} 1, \mathrm{O} 2, \mathrm{O} 3, \ldots \mathrm{O} 11$, (Fig. 6) are rotational and based on data from Table 4, it follows the Matrices A1, A2, A3, .., A11:

Transformations matrices of the End-effector coordinate frame (xe, ye, ze) to robot base frame $(\mathrm{x} 0, \mathrm{y} 0, \mathrm{z} 0)$ are:

$$
T_{1-11}=A_{1} \cdot A_{2} \cdot A_{3} \cdot A_{4} \cdot A_{5} \cdot A_{6} \cdot A_{7} \cdot \ldots A_{8} \cdot A_{9} \cdot A_{10} \cdot A_{11}=\left[\begin{array}{cccc}
c_{11} & c_{12} & c_{13} & d_{x} \\
c_{21} & c_{22} & c_{23} & d_{y} \\
c_{31} & c_{32} & c_{33} & d_{z} \\
c_{41} & c_{c 42} & c_{c 43} & c_{44}
\end{array}\right]
$$

The following variables $c_{11}$ to $c_{44}$ are given, where $c\left(\theta_{i}\right)=\cos \left(\theta_{i}\right)$ and $s\left(\theta_{i}\right)=\sin \left(\theta_{i}\right)$, for $i=1,2, \cdots, 11$. Using the D-H on above matrices (21) and after multiplying of these matrices the c41, c42, c43, and c44 are calculated.

$$
\left.c_{41}=0 \quad c_{42}=0 \quad c_{43}=0 \quad c_{44}=1\right\}
$$

Position x of End-effector is to (23): 


$$
d_{x}=\left|\begin{array}{c}
26 \cdot c\left(\theta_{9}\right) \cdot\left(s\left(\theta_{6}\right) \cdot s\left(\theta_{8}\right)-c\left(\theta_{6}\right) \cdot c\left(\theta_{7}\right) \cdot c\left(\theta_{8}\right)\right) \cdot\left[c ( \theta _ { 9 } ) \cdot \left(c\left(\theta_{8}\right) \cdot s\left(\theta_{6}\right)+c\left(\theta_{6}\right) \cdot\right.\right. \\
\left.\left.c\left(\theta_{7}\right) \cdot s\left(\theta_{8}\right)\right)-s\left(\theta_{9}\right) \cdot s\left(\theta_{6}\right) \cdot s\left(\theta_{8}\right)-c\left(\theta_{6}\right) \cdot c\left(\theta_{7}\right) \cdot c\left(\theta_{8}\right)\right] \cdot\left[30 \cdot s\left(\theta_{1}\right)+a_{2} \cdot c\left(\theta_{1}\right)\right. \\
s\left(\theta_{2}\right)+c\left(\theta_{2}\right) \cdot s\left(\theta_{1}\right)-11.4 \cdot s\left(\theta_{5}\right) \cdot\left[c\left(\theta_{3}\right) \cdot\left(c\left(\theta_{1}\right) \cdot c\left(\theta_{2}\right)-s\left(\theta_{1}\right) \cdot s\left(\theta_{2}\right)\right)+s\left(\theta_{3}\right) \cdot\right. \\
\left.\left(c\left(\theta_{1}\right) \cdot c\left(\theta_{2}\right)-s\left(\theta_{1}\right) \cdot s\left(\theta_{2}\right)\right)\right]+11.4 \cdot c\left(\theta_{4}\right) \cdot c\left(\theta_{5}\right) \cdot\left[c\left(\theta_{3}\right) \cdot\left(s\left(\theta_{1}\right) \cdot s\left(\theta_{2}\right)\right]\right.
\end{array}\right|
$$

Position $y$ of End-effector is to (24):

$$
d_{y}=\left|\begin{array}{c}
{\left[c ( \theta _ { 9 } ) \cdot \left(c\left(\theta_{6}\right) \cdot s\left(\theta_{8}\right)+c\left(\theta_{7}\right) \cdot c\left(\theta_{8}\right) \cdot s\left(\theta_{6}\right)+c\left(\theta_{6}\right) \cdot c\left(\theta_{8}\right)-c\left(\theta_{7}\right) \cdot s\left(\theta_{6}\right) \cdot\right.\right.} \\
\left.\left.s\left(\theta_{8}\right)\right)\right] \cdot\left[26 \cdot c\left(\theta_{1}\right)+30 \cdot c\left(\theta_{1}\right) \cdot c\left(\theta_{2}\right)-30 \cdot s\left(\theta_{1}\right) \cdot s\left(\theta_{2}\right)+11.4 \cdot s\left(\theta_{5}\right) \cdot\left[c\left(\theta_{3}\right)\right.\right. \\
\left(c\left(\theta_{1}\right) \cdot s\left(\theta_{2}\right)+c\left(\theta_{2}\right) \cdot s\left(\theta_{1}\right)-s\left(\theta_{3}\right) \cdot\left(c\left(\theta_{1}\right) \cdot c\left(\theta_{2}\right)-s\left(\theta_{1}\right) \cdot s\left(\theta_{2}\right)\right)\right]+11.4 \cdot \\
c\left(\theta_{4}\right) \cdot c\left(\theta_{5}\right) \cdot\left[c\left(\theta_{3}\right) \cdot\left(c\left(\theta_{1}\right) \cdot c\left(\theta_{2}\right)-s\left(\theta_{1}\right) \cdot s\left(\theta_{2}\right)\right)+s\left(\theta_{3}\right) \cdot\left(c\left(\theta_{1}\right) \cdot\right.\right. \\
\left.s\left(\theta_{2}\right)+\left(c\left(\theta_{2}\right) \cdot s\left(\theta_{1}\right)\right)\right]+c\left(\theta_{9}\right) \cdot\left(c\left(\theta_{6}\right) \cdot c\left(\theta_{8}\right)\right.
\end{array}\right|
$$

Position $z$ of End-effector is according to (25):

$$
d_{z}=\left|\begin{array}{c}
15 \cdot c\left(\theta_{7}\right)+c\left(\theta_{7}\right) \cdot\left(11.4 \cdot c\left(\theta_{5}\right) \cdot c\left(\theta_{4}\right)+10\right)+\left(c\left(\theta_{8}\right) \cdot c\left(\theta_{9}\right) \cdot s\left(\theta_{7}\right)-s\left(\theta_{7}\right)\right. \\
\left.s\left(\theta_{8}\right) \cdot s\left(\theta_{9}\right)\right) \cdot\left[26 \cdot c\left(\theta_{1}\right)+30 \cdot c\left(\theta_{1}\right) \cdot c\left(\theta_{2}\right)-30 \cdot s\left(\theta_{1}\right) \cdot s\left(\theta_{2}\right)+11.4 \cdot s\left(\theta_{5}\right)\right. \\
{\left[c\left(\theta_{3}\right) \cdot\left(c\left(\theta_{1}\right) \cdot s\left(\theta_{2}\right)+c\left(\theta_{2}\right) \cdot s\left(\theta_{1}\right)-s\left(\theta_{3}\right) \cdot\left(c\left(\theta_{1}\right) \cdot c\left(\theta_{2}\right)-s\left(\theta_{1}\right) \cdot s\left(\theta_{2}\right)\right)\right]\right.} \\
+11.4 \cdot c\left(\theta_{4}\right) \cdot c\left(\theta_{5}\right) \cdot\left[c\left(\theta_{3}\right) \cdot\left(c\left(\theta_{1}\right) \cdot c\left(\theta_{2}\right)-s\left(\theta_{1}\right) \cdot s\left(\theta_{2}\right)\right)+s\left(\theta_{3}\right) \cdot\right. \\
\left.\left(c\left(\theta_{1}\right) \cdot s\left(\theta_{2}\right)+\left(c\left(\theta_{2}\right) \cdot s\left(\theta_{1}\right)\right)\right]\right]-c\left(\theta_{8}\right) \cdot s\left(\theta_{7}\right) \cdot s\left(\theta_{9}\right)
\end{array}\right|
$$

The initial position is defined as follows (Fig.7a):

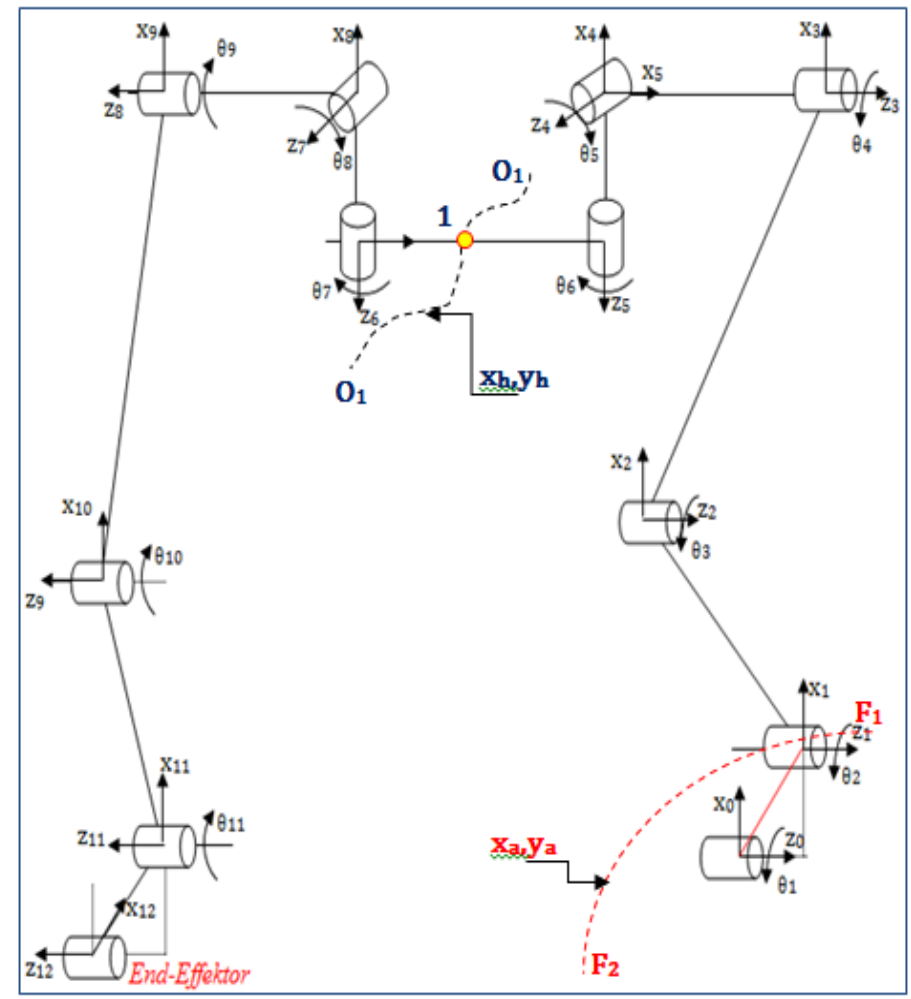

Fig. 7a Initial position

Where: $x_{a 1}$ and $x_{h}$ are 


$$
\begin{aligned}
& x_{a 1}=\left|\begin{array}{c}
x_{a}(t, k) \text { if } 0<t \leq s \\
x_{a}(s, k) \text { if } s<t \leq 2 s+2 d \\
x_{a}[t-(2 s+2 d), 2 k]+x_{a 1}(2 s+2 d)-x_{a}(0,2 k) \text { if } 2 s+d<t \leq 3 s+2 d
\end{array}\right| \\
& x_{h}=\left|\begin{array}{c}
x_{h s}(t, k) \text { if } 0<t \leq s \\
x_{h D}(t, k) \text { if } s<t \leq s+d \\
x_{h s}[t-(s+d), 2 k]+x_{h}(s+d)-x_{h s}(0,2 k) \text { if } s+d<t \leq 2 s+d \\
x_{h D}[t-(s+d), 2 k]+x_{h}(1.3)-x_{h D}(s, 2 k) \text { if } 2 s+d<t \leq 2 s+2 d \\
x_{h s}[t-(2 s+2 d+0.01), 3 k]+x_{h}(2 s+2 d)-x_{h D}(0,3 k) \text { if } 2 s+2 d<t \leq 3 s+2 d
\end{array}\right|
\end{aligned}
$$

From equations (22), (23), (24), (25) and (27) follows for the angle $\theta_{4}(28)$.

$$
\theta(t)_{4 l e f t-h i p}=a \cos \left(\frac{\left(x_{h}-x_{a 1}\right)-l_{1} \cdot \cos \left(\theta_{1}\right)}{l_{2}}\right)
$$

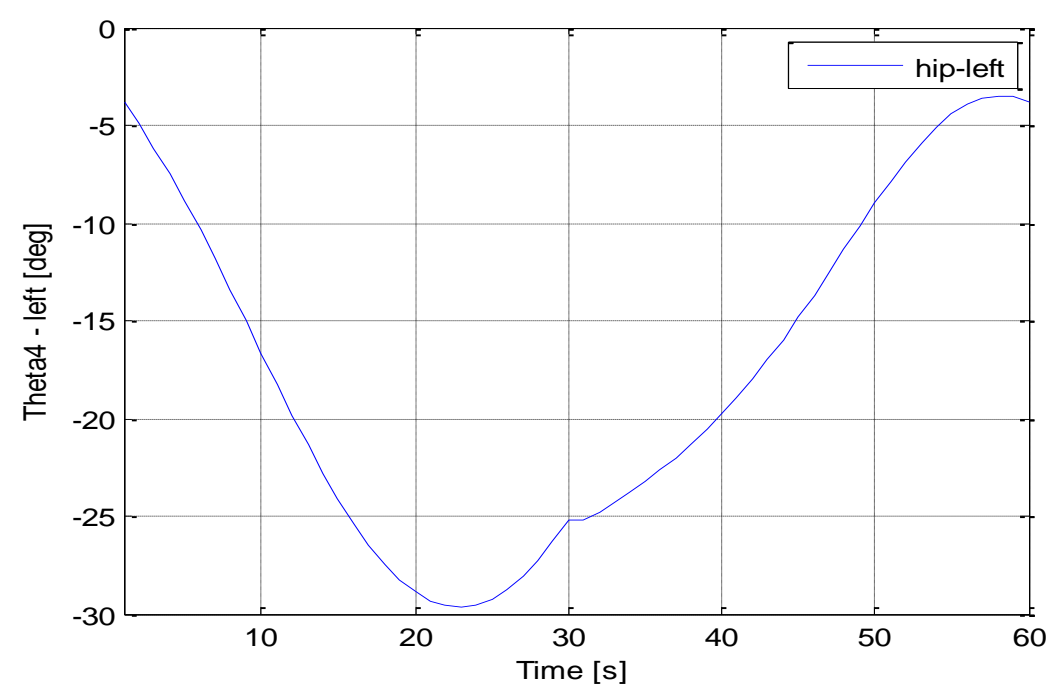

Fig. 7b Joint angle; hip left for two steps

$$
\left|\begin{array}{c}
O_{1}=x_{h}-x_{a 1}, \quad O_{2}=x_{h}-x_{a 2} \\
F_{1}=y_{h}-y_{a 1}, \quad F_{2}=y_{h}-y_{a 2} \\
E_{1}=\left(\frac{\left(x_{h}-x_{a 2}\right)^{2}+l_{1}^{2}+\left(y_{h}-y_{a 1}\right)^{2}-l_{2}^{2}}{2 l_{1}^{2}}\right)
\end{array}\right|
$$

Where: $x a 2, y a 1$ and $y a 2$ are 


$$
\begin{aligned}
& x_{a 2}=\left|\begin{array}{c}
0 \text { if } \quad 0<t \leq s+d \\
x_{a}[t-(s+d), 2 k]-x_{a}(0,2 k] \quad \text { if } \quad s+d<t \leq 2 s+d \\
x_{a 2}(2 s+d) \text { if } \quad 2 s+d<t \leq 3 s+3 d
\end{array}\right| \\
& y_{a 1}=\left|\begin{array}{c}
y_{a}(t, k) \text { if } \quad 0<t \leq s \\
0 \text { if } s<t \leq 2 s+2 d \\
y_{a}[t-(2 s+2 d), 2 k] \text { if } \quad 2 s+2 d<t \leq 3 s+2 d
\end{array}\right| \\
& y_{a 2}=\left|\begin{array}{c}
0 \text { if } 0<t \leq s+d \\
y_{a}[t-(s+d), 2 k] \quad \text { if } \quad s+d<t \leq 2 s+d \\
0 \text { if } 2 s+d<t \leq 3 s+3 d
\end{array}\right|
\end{aligned}
$$

According to Fig. 10 and equations (31) and (29), the angle $\theta_{2}$ can be calculated (34).

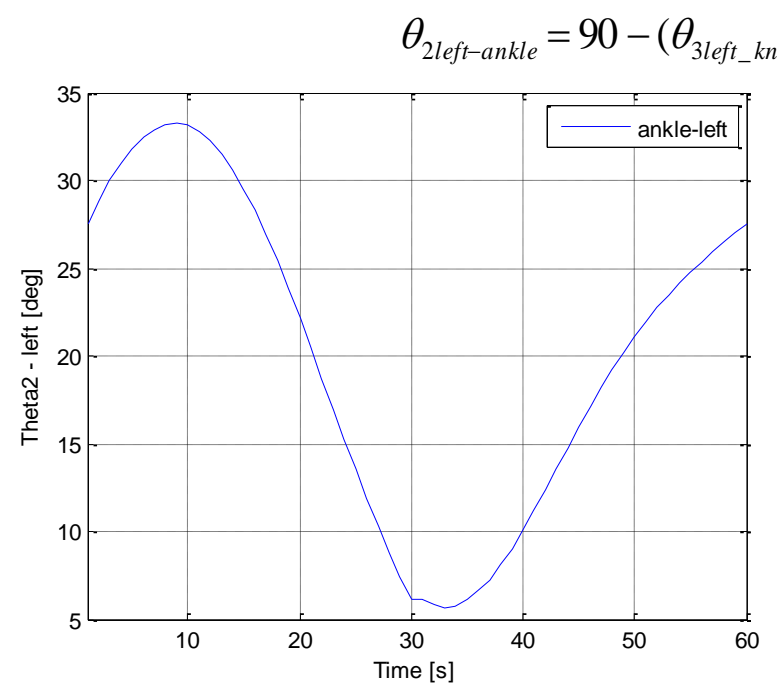

Fig. 8 Joint angle; ankle left for two steps

$$
\left.+\theta_{4 l e f t \_h i p}\right)
$$

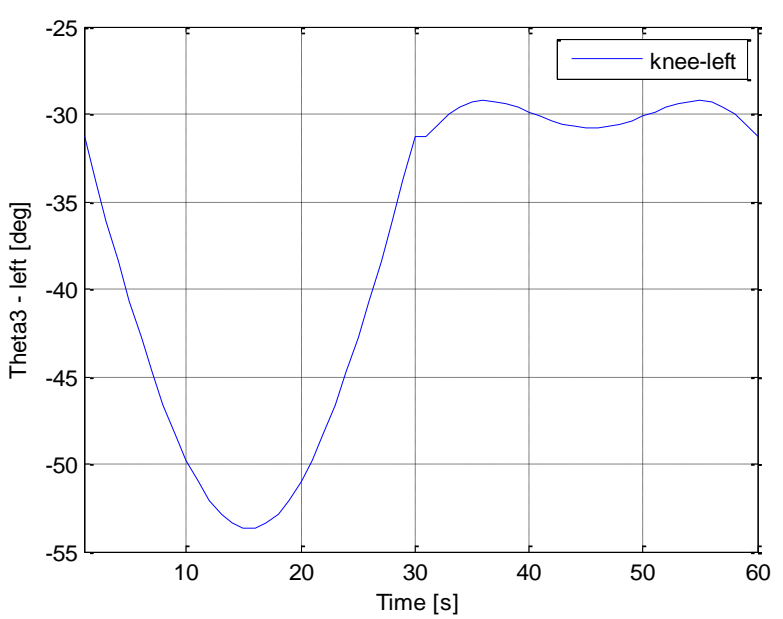

Fig. 9 Joint angle; knee left for two steps

From Fig.10 and the equations we can obtain angle $\theta_{11}$ as follows (35).

$$
\theta_{11 \text { Rightankle }}=90-\left(\theta_{9 \text { Righthip }}+\theta_{10 \text { Rightknee }}\right)
$$

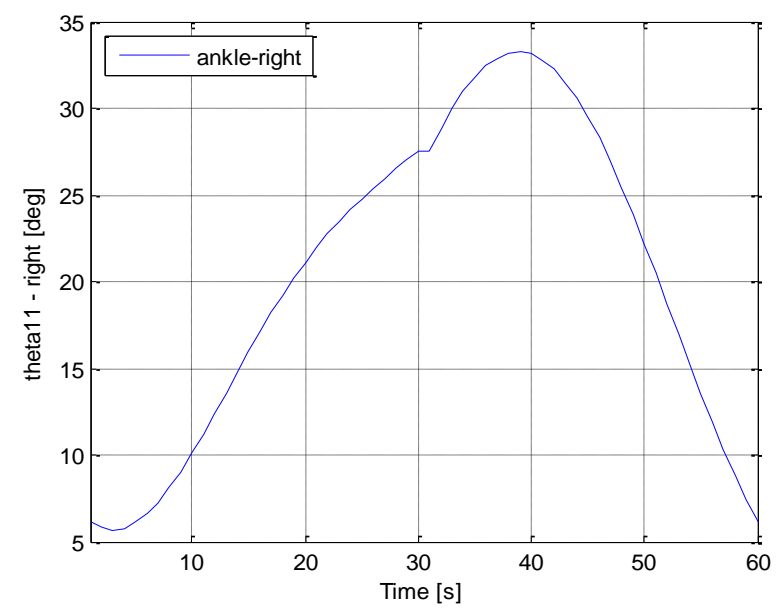

Fig. 10 Joint angle; ankle right for two steps

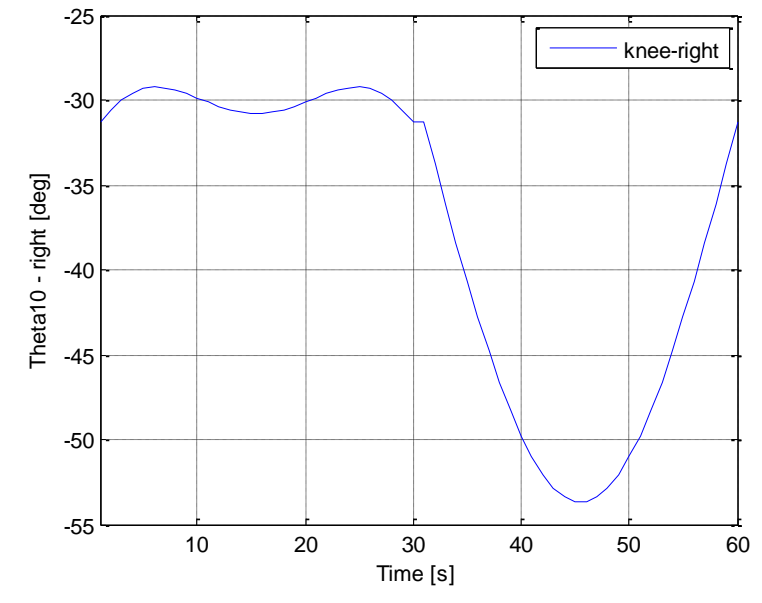

Fig. 11 Joint angle; knee right for two steps 


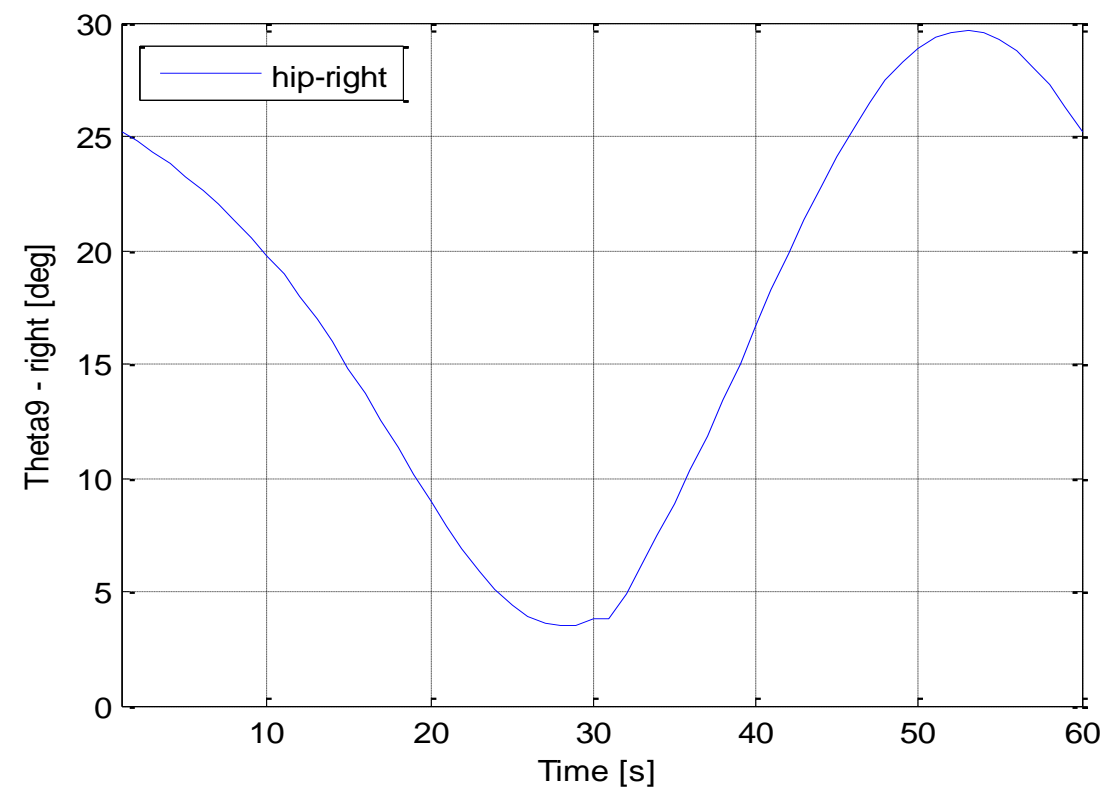

Fig. 12 Joint angles; hip right for two steps

Now all the angles for the joint are calculated and, therefore, it is possible to determine the position and orientation of the right/left hip. The kinematic model is derived such that the global positions, velocity, and acceleration of each link.

\section{Stability analysis of humanoid walking}

In this model it is assumed that the mass of the robot can be lumped to the robot's centre of mass and the legs of the robot are assumed to be mass. The following discussion is based on the research performed by professor Kajita [1], [6], [11], [15].

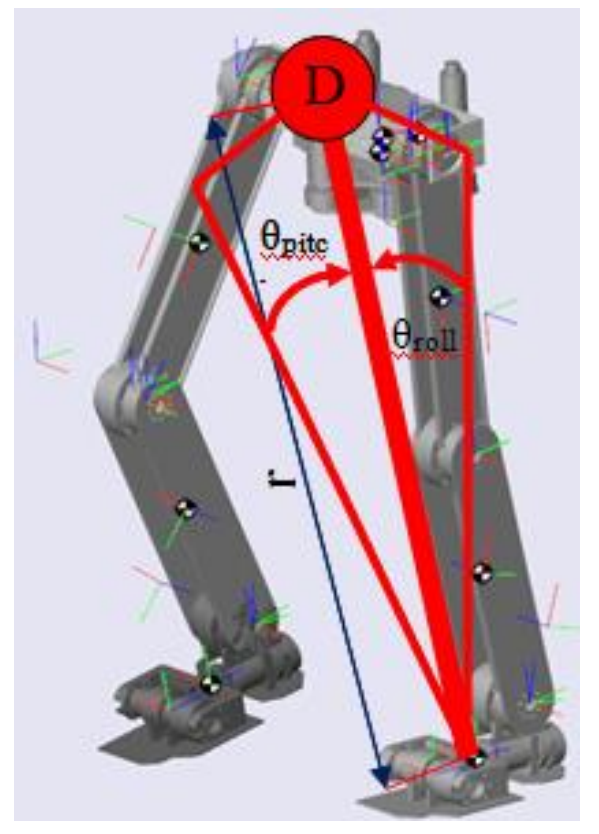

Fig. 13 Inverted pendulum [2], [4], [13]

The position of the mass point $\mathrm{D}$ is specified by a set of state variables, $\left(\theta_{\text {pitch }}, \theta_{\text {roll }}, r\right)$, which are related to the Cartesian coordinates by: 


$$
\left.\begin{array}{l}
x=r \cdot \sin \left(\theta_{\text {pitch }}\right), \quad y=-r \cdot \sin \left(\theta_{\text {roll }}\right) \\
z=r \cdot \sqrt{1-\left(\sin \left(\theta_{\text {pitch }}\right)\right)^{2}+\left(\sin \left(\theta_{\text {roll }}\right)\right)^{2}}
\end{array}\right\}
$$

If $\tau_{\text {roll }}, \tau_{\text {roll }}$ and $f$ are the actuator torque and force associated with these state variables, then the equation of motion is given by:

$$
m \cdot\left[\begin{array}{l}
\ddot{x} \\
\ddot{y} \\
\ddot{z}
\end{array}\right]=\left(\mathfrak{J}^{T}\right)^{-1} \cdot\left[\begin{array}{l}
\tau_{\text {roll }} \\
\tau_{\text {pitch }} \\
f
\end{array}\right]+\left[\begin{array}{l}
0 \\
0 \\
-m g
\end{array}\right]
$$

In the above equation, the Jacobian is given by:

$$
\left.\mathfrak{I}=\frac{\partial p}{\partial q}=\left[\begin{array}{ccc}
0 & r \cdot \cos \left(\theta_{\text {pitch }}\right) & \sin \left(\theta_{\text {pitch }}\right) \\
-r \cdot \cos \left(\theta_{\text {roll }}\right) & 0 & -\sin \left(\theta_{\text {roll }}\right) \\
\frac{-r \cdot \cos \left(\theta_{\text {roll }}\right) \cdot \sin \left(\theta_{\text {roll }}\right)}{D} & \frac{-r \cdot \cos \left(\theta_{\text {pitch }}\right) \cdot \sin \left(\theta_{\text {pitch }}\right)}{D} & D
\end{array}\right]\right\}
$$

The following equation can then be derived for the dynamics of the pendulum:

$$
\left.\begin{array}{l}
m \cdot(-z \cdot \ddot{y}+y \cdot \ddot{z})=\tau_{\text {roll }} \cdot \frac{D}{\cos \left(\theta_{\text {roll }}\right)}-m g \cdot y \\
m \cdot(-z \cdot \ddot{x}+x \cdot \ddot{z})=\tau_{\text {pitch }} \cdot \frac{D}{\cos \left(\theta_{\text {pitch }}\right)}-m g \cdot x
\end{array}\right\}
$$

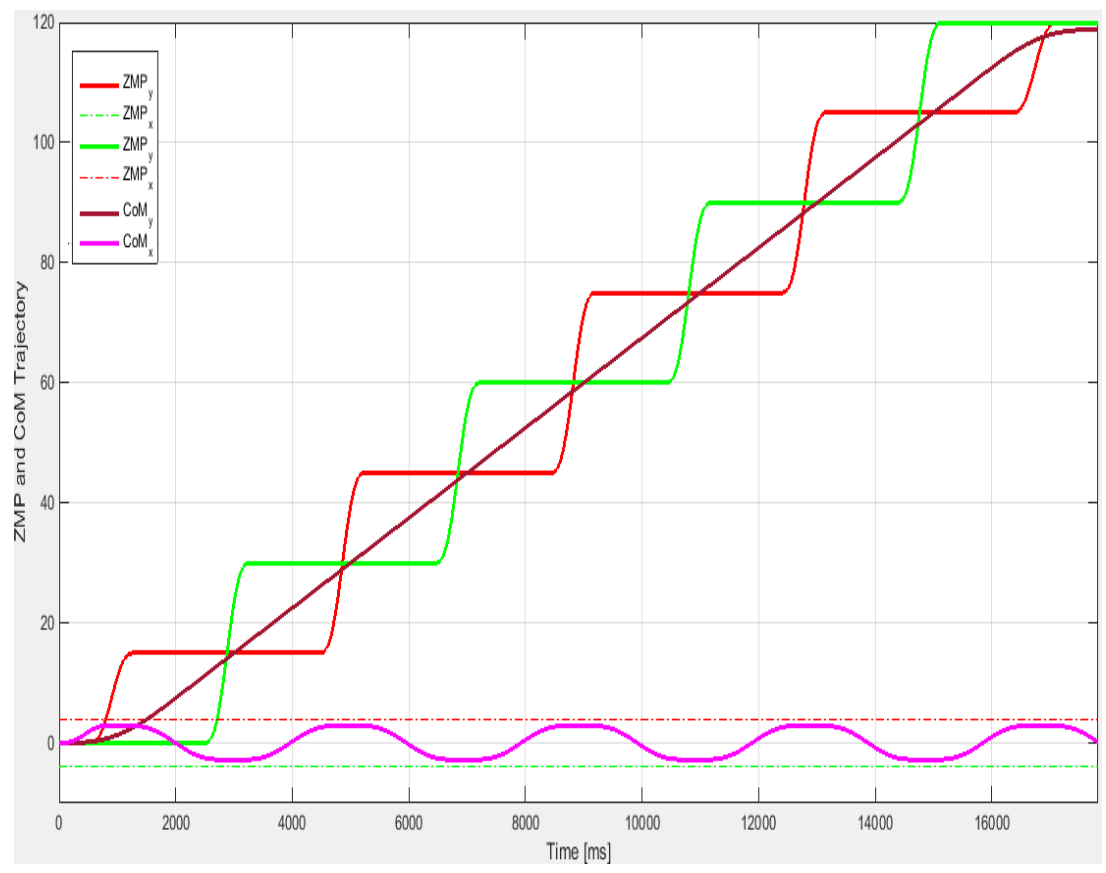

Fig. 14 ZMP and CoG trajectory in oxy - direction [2], [6] 


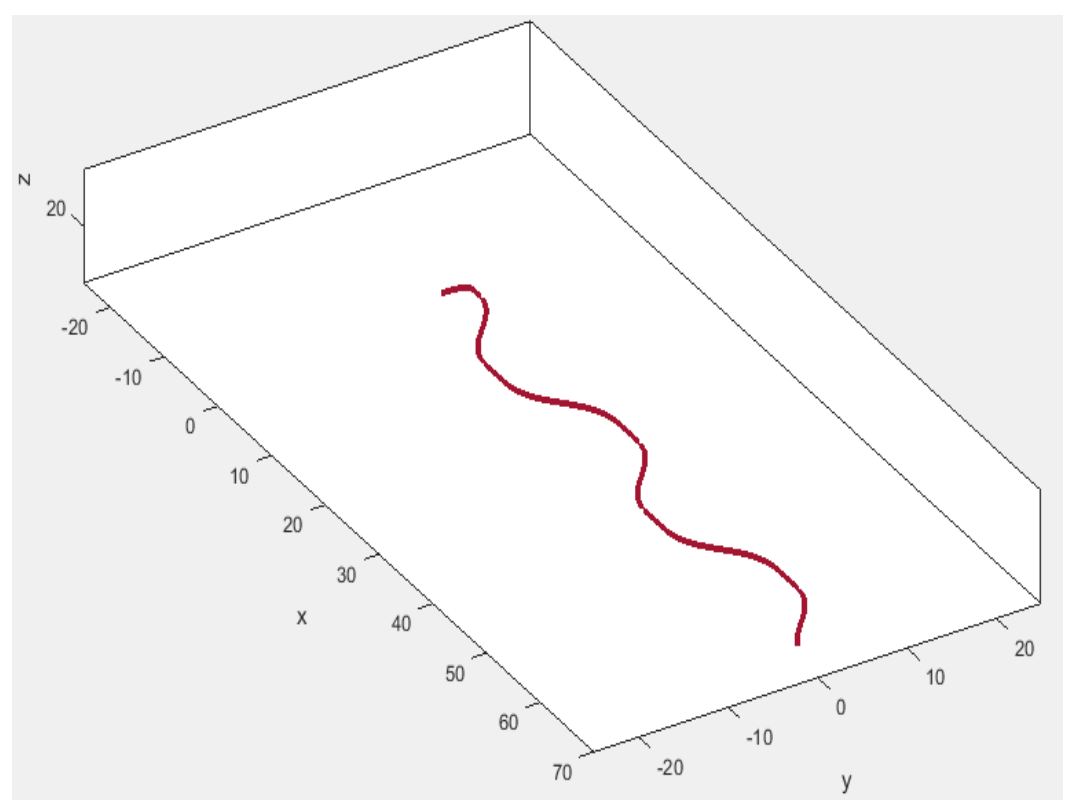

Fig. 15 Cog trajectory in oxyz

\section{Robot Simulations}

The simulator used for the robot is developed in the SimMechanics Toolbox of Matlab/Simulink [17]. The simulator is prepared in order to develop the motions and predict the actual results before applying them to the real robot. The model in $3 \mathrm{D}$ was built using the already designed model for the double foot. For that matter, it was necessary to allow motion and calculate the forces in the third dimension. Further, the humanoid robot must be composed of two legs, add the physics of the main body, and implement the hip abduction joints between the main body and the legs [2].

\section{Modelling of the floor}

The problem with the SimMechanics toolbox was the lack of object collision, which is crucial for the simulation as the normal and frictional forces between the floor and the robot are the key for gait. To solve this problem, the floor was modelled as a PD controller. This controller exerted normal forces on a few selected points of the robot sole, only when these points were on or below the floor [2], [3], [7].

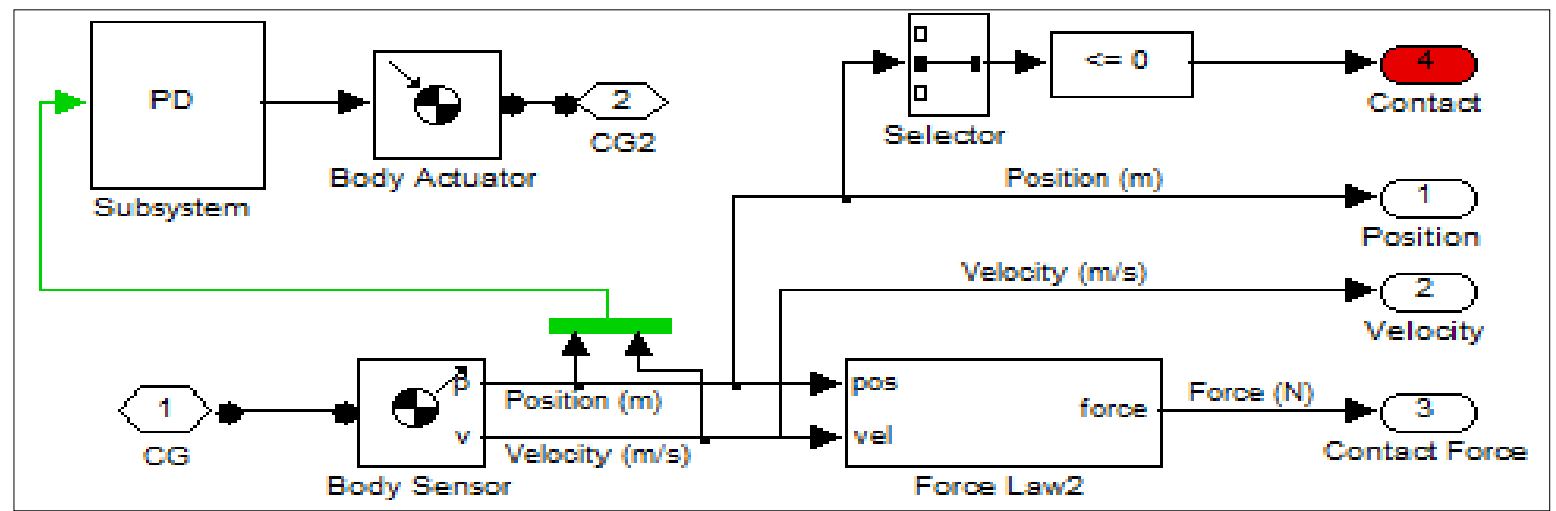

Fig. 16 Ground Contact

The error input of this PD controller, as shown in Fig. 15, was the deviation of these points from the floor level. High proportional and derivative gains were used to minimise the sinking 
of the feet on the floor. The floor also exerted viscous friction forces when the feet were in contact with the floor. Fig. 17 and Fig. 18 (xy - direction) show the three continually step positions of robot left leg. During the period when the left leg is on the ground, the right leg is in the air (up) [2], [3].

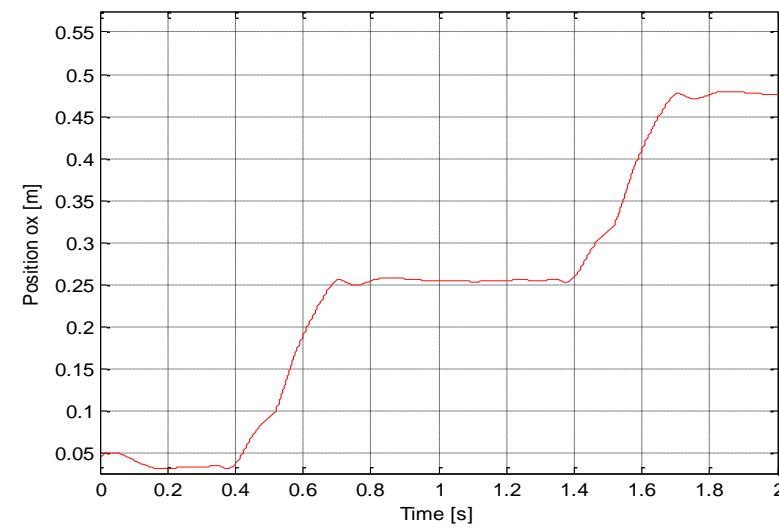

Fig. 17 Position (x) of the left foot

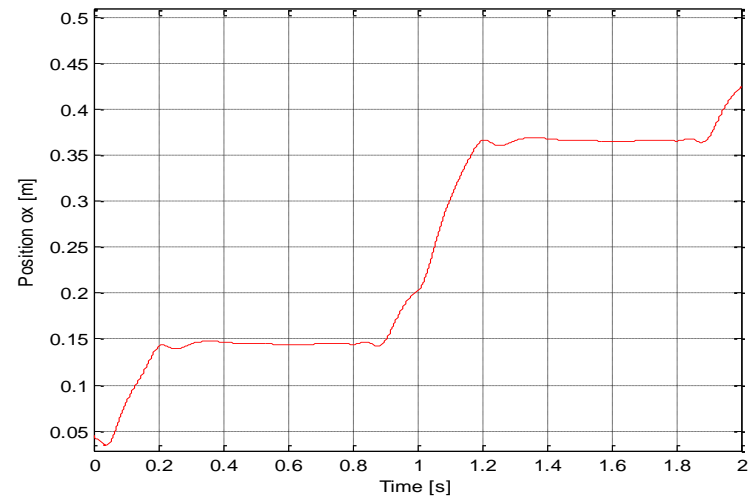

Fig. 19 Position (x) of the right foot

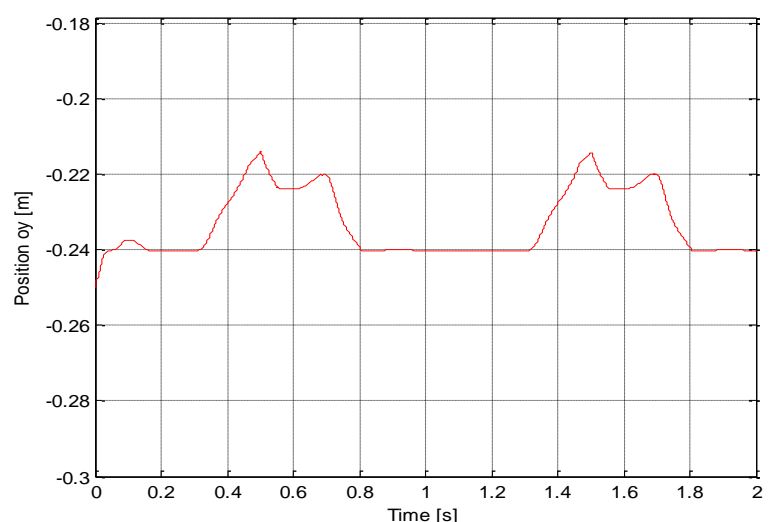

Fig. 18 Position (y) of the left foot

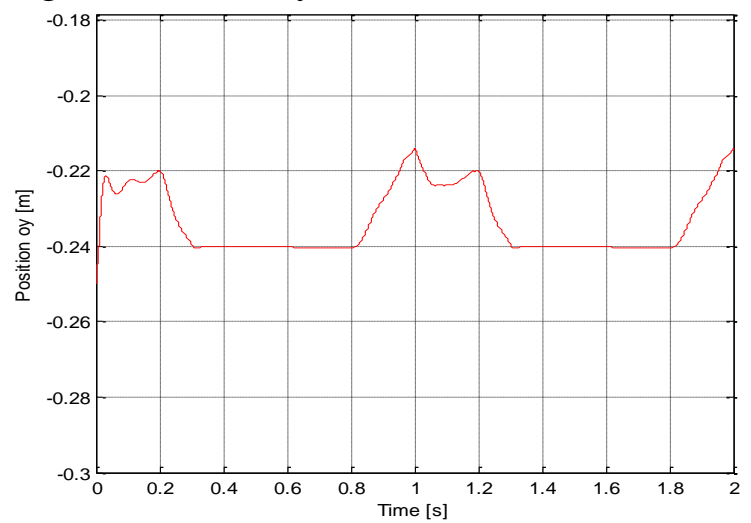

Fig. 20 Position (x) of the right foot

\section{CONCLUSIONS}

The main goal of this paper was to derive the walking model for dynamic behaviour of a humanoid robot. For two different locomotion phases a new approach was created. The model of robot (only lower body), as presented in this paper, has 11 DOF's. The modelling of a humanoid robot is described and presented. We have considered that walking and the stability of a humanoid robot are very important tasks for research and analysis. Our method can be applied to a wide range of step lengths, since the new kinematic, dynamic, ZMP, and path recognizing approaches, which have been developed, are presented in this work. A new kinematic model for a humanoid robot is built on trajectory calculations, which are based on mathematical equations. The kinematic model is a very efficient way to calculate the joint for ankles, hip, and knee. These calculations show the range of allowed positions for different angles, which should lead to the stability of the robot. The limitation on legs during the walking process, in other words with limitation of the body that connects the part of the hip to the foot which does not allow to open part (leg) back, is no more than 7.5 degrees. This means that this problem affects stability. We propose to increase this limit up to 15 degrees. A new dynamic model performs the calculation of force and torque for the humanoid robot's lower body. The obtained results show a real case scenario for the walking process of a robot. In this research it was further shown that actual servo and joint-motors have sufficient power to handle with robot 
stability. Also, two different trajectories (hip and ankle) are presented in this paper. Finally, the described dynamic, ZMP, and path recognizing approaches, which have been presented in this paper, have shown that the stability of humanoid robot during walking process is reached.

\section{REFERENCES}

[1] Sh. Kajita, et al. Humanoid Walking Pattern Generation by using Preview Control of Zero-Moment Point. Proceedings IEEE Conference on Robotics \& Automation Taipei, Taiwan, 2003, 14 - 19/09, 1620 - 1626.

[2] Xh. Bajrami. Dynamic modeling and simulation of a humanoid robot. PhD thesis.Vienna University of Technology, Austria 2013

[3] X. Bajrami, P. Kopacek, A. Shala, R. Likaj. Modeling and control of a humanoid robot. $e$ \& $i$ Elektrotechnik und Informationstechnik 2013 (130), No. 2, 61 - 66.

[4] P. Kopacek. Cost oriented humanoid robot. In Proceedings of the 18th IFAC world congress, Milano, Italy, 28 August - 2 September 2011, 2011

[5] G. Capi et al. Real time gait generation for autonomous humanoid robots: A case study for walking, Robotics and Autonomous Systems 2003 (42), 107 - 116.

[6] M. Omer, G. Reza, L. Hun-ok, T. Atsuo. SemiPassive Dynamic Walking for Biped Walking Robot Using Controllable Joint Stiffness Based on Dynamic Simulation. Japan: 2009 IEEE/ASME, July 14 - 17, 2009.

[7] X. Bajrami, et al. Trajectory Planning and Inverse Kinematics Solver for Real Biped Robot with 10 DOF-s., IFAC-PapersOnLine 49.29 2016, 88 - 93.

[8] Xh. Bajrami, et al. Kinematics and dynamics modelling of the biped robot. IFAC Proceedings Volumes 46.8 2013, 69 - 73.

[9] S. H. Hyon, D. Suewaka, Y. Torii, N. Oku. Design and Experimental Evaluation of a Fast Torque-Controlled Hydraulic Humanoid Robot. in IEEE/ASME Transactions on Mechatronics 2017 (22), No. 2, 623 - 634.

[10] P. Shahverdi, M. T. Masouleh. A simple and fast geometric kinematic solution for imitation of human arms by a NAO humanoid robot. 2016 4th International Conference on Robotics and Mechatronics (ICROM), Tehran 2016, 572 - 577.

[11] C. Goldbeck, L. Kaul, N. Vahrenkamp, F. Worgotter, T. Asfour, J. M. Braun. Two ways of walking: Contrasting a reflexive neuro-controller and a LIP-based ZMP-controller on the humanoid robot ARMAR-4. 2016 IEEE-RAS 16th International Conference on Humanoid Robots (Humanoids), Cancun, 2016, 966 - 972.

[12] X. Mu., Q. Wu. Synthesis of a complete sagittal gait cycle for a five-link biped robot. Robotica 2004 (21), 581 - 587.

[13] A. Byagowi, A Control System for a Humanoid Robot. PhD. Thesis Vienna University of Technlogy 2010.

[14] X. Bajrami et al. Genetic and Fuzzy logic algorithms for robot path finding. 5th Mediterranean Conference on Embedded Computing (MECO) 2016, 195 - 199. 
[15] Goldbeck, C., Kaul, L., Vahrenkamp, N., Worgotter, F., Asfour, T., \& Braun, J. M. (2016, November). Two ways of walking: Contrasting a reflexive neuro-controller and a LIPbased ZMP-controller on the humanoid robot ARMAR-4. In Humanoid Robots (Humanoids), 2016 IEEE-RAS 16th International Conference on (pp. 966 - 972). IEEE

[16] I. Virgala, T. Lipták, L. Miková. Snake Robot Locomotion Patterns for Straight and Curved Pipe. Journal of Mechanical Engineering - Strojnícky časopis 2018 (68), No. 2, $91-104$.

[17] M. Kelemen, A. Gmiterko, D. Hroncová. Simulation of Worm-Like Machine. Journal of Mechanical Engineering - Strojnicky časopis 2018 (68), No. 2, 25 - 34. 